\title{
Knowledge, attitude and practices towards blood donation in Pakistan: A nationwide survey
}

\begin{abstract}
Introduction: In Pakistan, the blood donation and transfusion practices are based on primitive practice of replacement donors whereas all around the world, the coherent practice of voluntary blood donation has replaced the replacement donation. Pakistan, as a signatory to the World Health Organization (WHO) resolution has thus envisioned reaching $100 \%$ Voluntary Non Remunerated Blood Donation (VNRBD) by the year 2020 .

Material and methods: The study was conducted to understand the current state of affairs of potential future donors with the objective of creating a directed Public Awareness Campaign (PAC). A cross-sectional, descriptive study was done and forms filled by 3,000 respondents from all over Pakistan. A pretested questionnaire form with 30 questions assessing the Knowledge, Attitude and Practice of the respondents was used. The results were analyzed by SPSS 17 .

Results: Out of the total participants, $49 \%$ were non donors and $22 \%$ were replacement donors. Main source of information on blood donation were friends and family, i.e. $65 \%$. Misconception regarding blood donation were rampant with $62 \%$ respondents believing that blood donation can result in transfusion transmitted infection. Negative attitude stemmed from fear of the procedure in $59 \%$ respondents. A majority of respondents, i.e. $84 \%$ had access to internet, $96 \%$ had mobile phones and $44 \%$ of respondents used social media in their leisure time. This resource can be tapped for reliable and effective public awareness campaign. The reason for donation was altruism in $56 \%$ cases and pressure from family and friends $28 \%$.
\end{abstract}

Conclusion: Misconceptions and fear against voluntary donation of blood need to be addressed via a reliable and accessible medium. The internet and social media seems promising for such a Public Awareness Campaign.

Keywords: blood, donation, voluntary, Pakistan, KAP
Volume I Issue 4 - 2015

\author{
Usman Waheed, Muneeba Azmat, Hasan \\ Abbas Zaheer \\ Safe Blood Transfusion Programme, Ministry of National Health
} Services, Pakistan

Correspondence: Usman Waheed, Safe Blood Transfusion Programme, Ministry of National Health Services, Government of Pakistan, Tel 0092-333-5638702, Fax 0092-5I-9263238, Email usman.waheed07@gmail.com

Received: October 25, 2015 | Published: November 09, 2015
Abbreviations: KAP, knowledge attitude practice; PAC, public awareness campaign; DAC, donor awareness campaign; TTI, transfusion transmitted infection; VNRBD, voluntary nonremunerated blood donor/donation; $\mathrm{BDO}$, blood donor organization

\section{Introduction}

The collection of blood from voluntary, non-remunerated blood donors is an important measure for ensuring the safety, quality, availability and accessibility of blood. The WHO recommends that all countries should be self-sufficient in all blood products and that every blood donation should be voluntary, anonymous and non-remunerated. World Health Assembly resolutions WHA 28.72 and WHA 58.13 urged member states to develop national blood transfusion services based on voluntary non-remunerated blood donation. ${ }^{1}$ Pakistan as a signatory to these resolutions has envisioned to reach $100 \%$ voluntary non remunerated blood donation till the year 2020 .

In Pakistan, the total numbers of blood centres are $1830^{2}$ with an annual estimated blood collection of 3.5 million. Currently the practice of voluntary blood donation in Pakistan is far from satisfactory. According to data collected in 2013 from 300 blood banks for WHO global database on blood safety, the estimated blood collection per 1,000 population is 16.6 , only $10.6 \%$ of people donate blood voluntarily while $88.3 \%$ blood donation is by family or friends or as replacement donation. ${ }^{3}$
Systems based on voluntary donation require effective organizational structures to facilitate community mobilization and the application of appropriate strategies for the motivation of safe donors from identified low risk groups of the populations. In countries with similar population pyramids as Pakistan, the young population has been identified as a key low risk donor group. Also statistical data on the prevalence of Transfusion Transmitted Infections (TTIs) in the donor population in the country and various reports on blood transfusion services during the last decade indicate that this population group provides blood donors during emergencies and disasters is accessible, educated, presents a high potential of becoming regular voluntary non remunerated blood donors. In countries still dependent on blood donation by patients' relatives or by paid donation, as is the case with Pakistan, an increase of the proportion of voluntary donors among blood donors will enhance safety and also help to shift the responsibility for blood provision from patients' relatives to the health care system itself.

This issue has been addressed in the Pakistan's new Blood Donor Policy ${ }^{4}$ for on-going centralization of hospital blood banking system towards the international model with a functional and spatial separation between centralized blood production and affiliated blood transfusion centres, a paradigm shift from replacement donation to voluntary blood donations. The adoption of the VNRBD concept has been recommended in the National Blood Policy and Strategic Framework (2014-20). ${ }^{5}$ This paradigm shift will require major 
efforts in community awareness building, for which it is important to understand existing levels of knowledge regarding blood donation and blood transfusion. Furthermore it is important to know the current attitude of people about blood donation, their perception of the need for donor blood, and to examine the basis of their impressions and decisions about participation or non-participation in this activity. Obtaining valid insights into the knowledge, attitudes and the practices $(K A P)$ related to blood and blood donation requires a representative nationwide study within a random sample from the target population.

The present Knowledge Attitudes and Practices (KAP) study assessed the knowledge, attitudes and practices regarding Blood Donation and determined the factors which influence donor and non-donor behavior. The KAP study also provided an insight into the motivations and misconceptions of our potential donors, the awareness of younger population, their aptitude and also their behavior pertaining to transfusion practices. The ultimate objective of the current study was to promote Voluntary Non Remunerated Blood Donation (VNRBD) through a Public Awareness Campaign (PAC) based on a thorough analysis of the Knowledge, Attitudes and Practices of a key segment of the society, i.e. college and university students at the age of 18-25years.

\section{Material and methods}

A cross-sectional, descriptive study was conducted over a period of three months (Jan-Mar 2012). The survey was conducted through university based Blood Donor Organizations (BDOs) working across the country inventory of which had already been prepared by the SBTP. ${ }^{6}$ In order to have a reliable sample size, multi-stage random cluster sampling approachwas followed in a total of 56 BDOs. The selected BDOs $(\mathrm{n}=20)$ then randomly selected donors and non-donors to participate in the study. 100 respondents were interviewed by each of the $20 \mathrm{BDOs}$ (2,000 forms). In addition, 1,000 questionnaire forms were collected from Armed Forces Institute of Transfusion (AFIT) and Pakistan Red Crescent Society (PRCS), two institutions with extensive experience in voluntary blood donation. The inclusion of the latter two organizations further generalized our study. The donors and non-donors were randomly selected by the BDO's in their respective operational domains that mostly included colleges, universities and institutes like AFIT and PRCS. The non-donors selected never donated blood and were member of the BDO's operational domain. The volunteering participants were interviewed by their respective BDO's after obtaining fully informed consent and assuring them of their confidentiality.

The questionnaire was designed with 30 questions (with closed ended multiple answers) to measure the KAP about blood donation, taking into account the respective social and cultural environment. The questionnaire had four sections having a variable number of questions with multiple options in each section.
i. socio-demographic characteristics;
ii. knowledge
iii. attitudes
iv. practices

The questionnaire was pre-tested in a pilot study in Islamabad/ Rawalpindi. The questionnaire was kept anonymous and each question included multiple options or statements. The raw data were "cleaned" and prepared for further processing. Statistical analysis was conducted by the assistance of Statistical Package for Social Sciences (SPSS) software version 17. Knowledge questions were analyzed differently from those in the Attitudes section, which in turn were analyzed differently from those in the Practices section.

\section{Results}

A total of 3,000 questionnaires were filled collected. The participants' average age was 21.84years. The results of sociodemographic characteristics are shown in Figure 1.

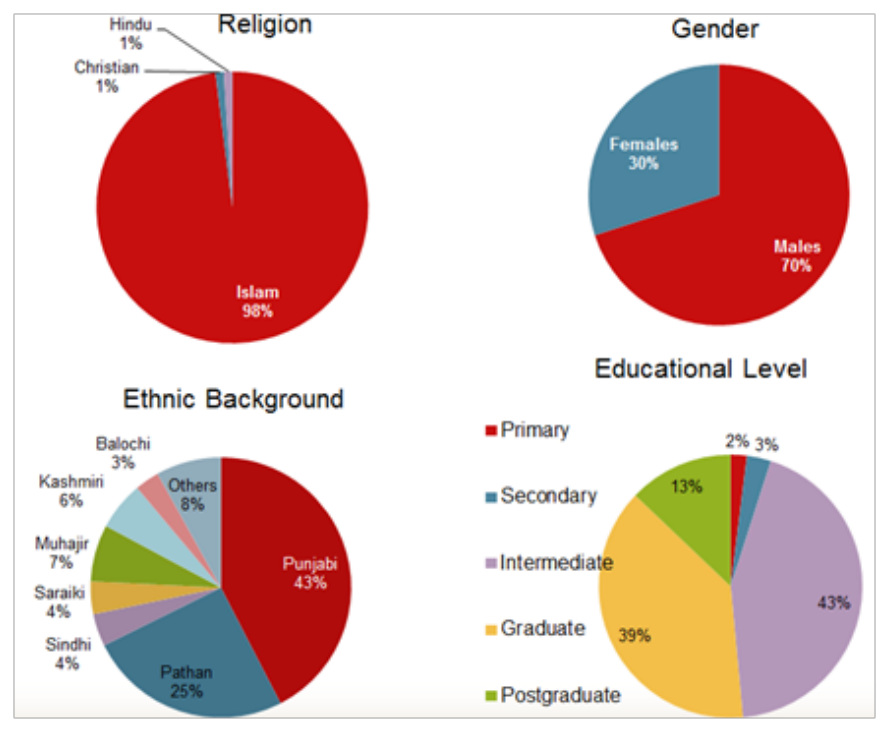

Figure I Socio-demographic characteristics of respondents.

Knowledge: The portion for knowledge was designed to assess the

i. Current source of knowledge on blood transfusion

ii. The knowledge gaps and misconception associated with blood donation practice and

iii. Potential source of information that can be used as building blocks for a future Public Awareness Campaigns (PAC).

The respondent's main source of information were family and friends in $65 \%$ of cases and social network in $28 \%$ of cases, $7 \%$ of the respondents had not received any information regarding blood donation practices. $56 \%$ of the respondents were in contact with blood donors, i.e. another source of information on blood donation. Around $27 \%$ respondents considered themselves donors. This percentage also included people who donated once and have stopped donating. The knowledge regarding criteria to be called a voluntary blood donor is lacking. When asked about any risks associated with blood donation, whopping $62 \%$ respondents responded that blood donation can result in contraction of blood borne disease, $25 \%$ responses considered blood donation causing faintness and $13 \%$ respondents considered blood donation a risk free process for the donor. Out of the total respondents, majority had access to internet/email and used cell phones giving an indication of easily available and potentially reliable and cheap source of information distribution for future PACs Figure 2.

Attitude: Under this section respondents were asked questions that assessed the reason for positive or negative attitude towards blood donation that could then be analyzed and the positive attitude could be reinforced and negative ones curbed. Positive attitude towards blood 
donation stemmed from altruism/the desire to help others in $56 \%$ of the case, other positive reinforcement included family and friends motivation (28\%) and religious organizations (16\%). The negative attitude towards the practice of blood donation was due to fear of the procedure and the concept that blood banks misuse/sell the blood products for monetary benefits Figure 3 .

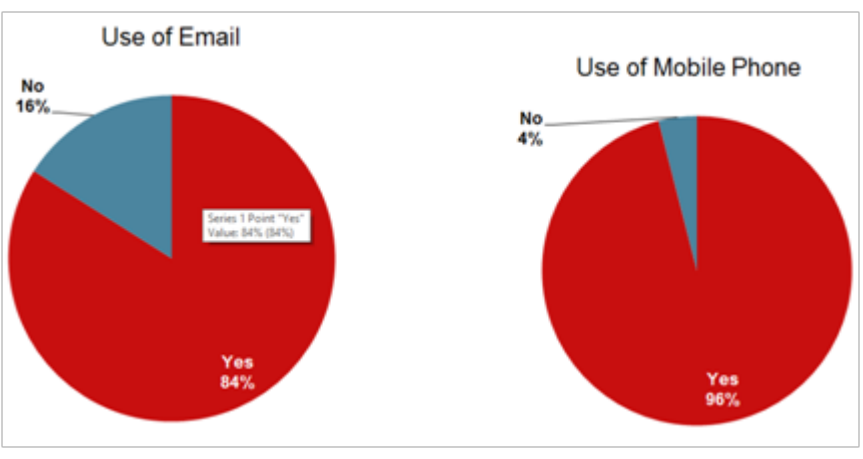

Figure 2 Restrictions for voluntary blood donation.

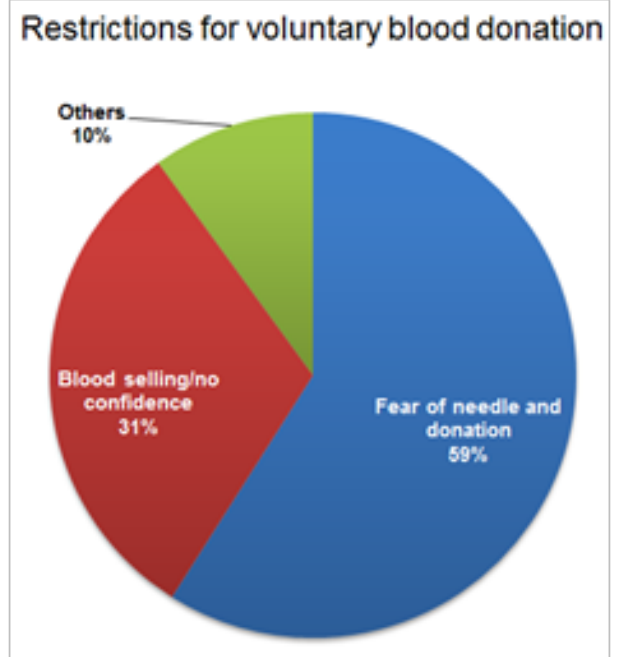

Figure 3 Restrictions for voluntary blood donation.

Practice: This segment highlighted the current practices that could potentially be used as future information distribution. Preferred activities of respondents in free time included sports, TV, reading and use of social network (Figure 4). Around 68\% respondents were actively involved in community services. $49 \%$ respondents had not ever donated blood, $29 \%$ had donated blood voluntarily and $22 \%$ had donated blood for friends and family.

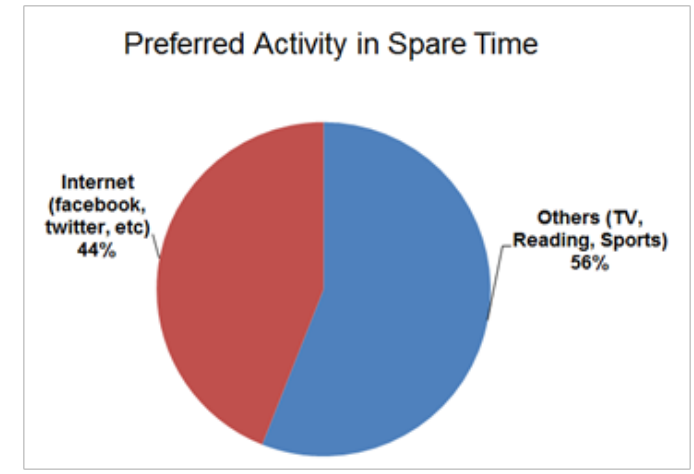

Figure 4 Preferred activity in spare time.

\section{Discussion}

There is so far very little published information and documentation about the background of blood donors and blood donation in Pakistan. Few small scale KAP studies have revealed a diverse set of myths and peculiar perceptions of public about blood donation in Pakistan. ${ }^{7}$ Females are about $50 \%$ of the total population but are completely under-represented in terms of donations collected. Another study among the health professionals showed that only $3.4 \%$ of doctors and $0 \%$ of paramedics were regular voluntary blood donors. ${ }^{8}$

The results from the current study showed that the adult male population has the major participation in the activity of blood donation. Female population that forms more than half of total country population was under represented in the blood donor pools. There is a great need for another in depth study that would explore the reasons for this discrepancy and any possible means to address this issue.

For development of a PAC (public awareness campaign) and DAC (donor awareness campaign), some valuable insights were achieved including the frequent and heavy use of electronic media and social network by the respondents in their spare time, however, it was not a major source of information to the respondents when it comes to blood donation practices. There were a few misconception and knowledge gaps prevailing among the population. Correct dissemination of information by reliable sources is required. The major source of information has to shift from family and friends to authentic sources.

Positive attitude towards blood donation was attributed to altruism and motivation by friends and family or blood donor organization. Another consequential finding is that the $56 \%$ of the study participants were in contact with blood donors. Through a structured DAC, these donors can be made a potential source of dissemination of correct knowledge to the non-donor population. Current blood donors can be used to motivate other non-donors, "donor brings another donor" policy.

In a country like Pakistan, where the individuals are under peer pressure mostly from their relatives, people rely on advices and comments conformed by their peers to form an opinion. A study conducted in 2009 highlighted the fact that relatives were paramount in molding the blood donation practices among the young ones ${ }^{8}$. Our study showed that relatives and friends also seem to be the informers regarding blood donation practices. This may lead to dissemination of incorrect information. The reliance of people on relatives can explain the prevalence of myths among the population regarding blood donation. ${ }^{9}$ The negative attitude towards the process including fear of the procedure and lack of trust stems from ignorance. Good awareness about the procedure, the need for it and transparency in the procedure is required to overcome these issues

The majority of Pakistani population is youngsters. As expected, our study showed the age of study group to fall between 20-25years with a mean age of 21.84years. The current study did not undertake an in-depth review of interplay between religion and blood transfusion practices (most respondents were Muslims). If we are to establish a focused campaign for public and donor awareness, the elemental role of religion cannot be stressed enough. Same is the case with ethnicity, the study showed a skewed representation of provincial population. Efforts to include equal representation in a country wide study or a divided area wise undertaking of KAP and implementation of consequential effective directed measures is required for optimal 
results. Most of the respondents were educated however the population education status is different in different areas of Pakistan, from $96 \%$ literacy rate of Islamabad to as low as $16 \%$ in areas of Baluchistan. KAP of illiterate population is bound to be different and difficult. Focus should be kept on the literate population for now, due to meagre resources and eventually it must be spread to include the illiterate population as well.

\section{Conclusion}

To overcome the negative attitude to VNRBD and reported fear, it is important to provide adequate information about donation to potential donors. Although answers on motivational and restrictive aspects of blood donation suggest the importance of family and friends, the reported preference for internet as leisure time activity suggests that internet has promising possibilities as main source of information in a Public Awareness Campaign, for which further study is needed. Both PAC and DAC measures would be required when taking important decisions, PAC would provide knowledge and create awareness about the importance of blood transfusion to the entire population. DAC measures would target more directly potential donor groups through more specific channels, including SMS, internet, etc.

\section{Acknowledgements}

None.

\section{Conflict of interest}

The author declares no conflict of interest.

\section{References}

1. The Melbourne Declaration on 100\% Voluntary Non-remunerated Donation of Blood and Blood Components. Geneva, Switzerland: World health organization; 2009.

2. Zaheer HA, Waheed U, Farhan Y. Adoption of WHO standard operating procedures for blood screening in hospitals of Islamabad, Pakistan. Afr $J$ Pharm Pharmacol. 2012;6(11)

3. WHO Global Database on Blood Safety. World health organization; 2013.

4. National blood donor policy. Safe blood transfusion programme, Ministry of national health services, Government of Pakistan; 2011.

5. National blood policy and strategic framework. Safe blood transfusion programme, Ministry of national health services, Government of Pakistan; 2014

6. Inventory of blood donor organizations. Safe blood transfusion programme, Ministry of national health services, Government of Pakistan; 2012

7. Mumtaz Z, Bowen S, Mumtaz R. Meanings of blood, bleeding and blood donations in Pakistan: implications for national vs global safe blood supply policies. Health Policy Plan. 2011. p. 1-9.

8. Gilani I, Kayani ZA, Atique M. Knowledge, attitude and practices (KAP) regarding blood donation prevalent in medical and paramedical personnel. J Coll Physicians Surg Pak. 2007;17(8):473-476.

9. Baig M, Habib H, Haji A, et al. Knowledge, Misconceptions and motivations towards blood donation among university students in KSA. Pak J Med Sci. 2013;29(6):1295-1299. 